\title{
Pre- and Intra-operative Planning and Simulation of Percutaneous Tumor Ablation
}

\author{
Torsten Butz ${ }^{1,2}$, Simon K. Warfield ${ }^{2}$, Kemal Tuncali ${ }^{3}$, Stuart G. Silverman ${ }^{3}$, \\ Eric van Sonnenberg ${ }^{3}$, Ferenc A. Jolesz ${ }^{2}$, and Ron Kikinis ${ }^{2}$ \\ 1 Swiss Federal Institute of Technology at Lausanne(EPFL), Signal Processing \\ Laboratoy (LTS), CH-1015 Lausanne, Switzerland \\ torsten.butz@epfl.ch, \\ http://ltswww.epfl.ch FAX: +41-21-693-7600 \\ 2 Surgical Planning Laboratory, Department of Radiology \\ 3 Department of Radiology, Brigham and Women's Hospital and Harvard Medical \\ School, 75 Francis Street, Boston, MA 02115, USA
}

\begin{abstract}
We developed a software tool for pre-operative simulation and planning, and intra-operative guidance, of minimally invasive tumor ablation, including radiofrequency-, laser- and cryo-therapy. This tool provides a pre- and intra-operative optimization of the treatment plan, in order to avoid dangerous probe trajectories, undertreatment of the tumor, and excessive ablation of healthy tissues.

The simulation is performed within a virtual operating-room consisting in essence of the patient's segmented anatomy from pre- or intraoperatively acquired MR scans. Virtual probes can be placed into this scene and at the formation of ablated tissue at their tips can be simulated. To verify the simulated treatment plans, we introduced an objective quality measure which also enables a semi-automated optimal probe placement.

To show the use and to underline the importance of our tool, we investigated a cryo-therapy case which did not succeed. We show that our software would have predicted the failure of the chosen treatment plan and how it could have increased the efficacy of the procedure.
\end{abstract}

\section{Introduction}

Recent developments in interventional imaging, such as interventional MRI [1, have opened a vast range of promising medical applications. For example, several techniques for minimally-invasive percutaneous tumor ablation have been developed; some relying on heating (focused ultrasound, laser or radiofrequency) and some on freezing (cryo-therapy) the tissue to kill the cells. The immune system then removes the ablated tissue from the body.

We have placed the emphasis of this paper upon cryo-therapy. Nevertheless, the generalization to other minimally-invasive ablation techniques is straight forward and some important aspects will be explained for both cryo- and radiofrequency-ablation. 


\subsection{Limitations of Conventional Planning}

In tumor ablation, the radiologists try to cover the arbitrarily shaped tumor with a given number of discrete objects of approximately known shape, representing the critical ablation temperatures for killing cancerous cells. In cryotherapy, the temperatures within the tumor should nowhere be above, and for thermo-ablation below, this bound. For interstitial tumor ablation, the cooling, respectively heating source is located on the tip of a needle-like probe which is percutaneously inserted through a catheter into the tumor.

The safety and success of a treatment plan depends mainly on the three following points:

1. Choosing secure probe trajectories.

2. Ablating all the cancerous cells.

3. Killing as little of the surrounding healthy tissue as possible.

Unfortunately it is very difficult to find the treatment plan that optimizes these three central factors when the radiologists have to rely only on 2D-slices of pre- and intra-operative MR-scans. In such data, the important 3D shapeinformation of the different anatomical objects (for example the tumor) and their mutual positions is not efficiently visualized.

\subsection{Improving the Planning Process}

The presented tool provides several features to improve the planning process for minimally-invasive percutaneous tumor ablation. Segmented MR scans enable a $3 \mathrm{D}$ view of the patient's anatomy and virtual needles visualize efficiently the probe's trajectories (optimize point 1 above). We also simulate and visualize the frozen tissue at the tip of the probes. An objective quality measure optimizes and classifies the simulated treatment plans according to point 2 and 3 above.

Results are presented in the context of liver cryo-therapy ([2]). We show how our tool can optimize secure probe trajectories and placements while avoiding undertreatment of the tumor.

\section{Methods}

The main functions of the tool are a $3 \mathrm{D}$ visualization of the patient's anatomy, a simulation of the probes and the frozen tissue, and a semi-automated optimization minimizing an objective quality measure to assess probe placement.

\subsection{Visualization}

To have a variety of visualization modes at the radiologist's disposal, we added our planning and simulation tool into an existing software package, called the $3 D$ Slicer ([3]). In this way, our planning tool can be used in conjunction with all the standard functionalities of that program. Let's shortly summarize its main features: 
- The visualized 2D slices of 3D MR scans can be arbitrarily oblique, or orthogonal and oriented relative to either the coordinate frame of the scanner or the tracked surgical instrument.

- Different MR scans (e.g. SPGR, T2-weighted MRI, etc.) can be registered and visualized simultaneously or separately.

- Surface models representing the patient's anatomy can be generated from segmentations of MR scans and visualized in a 3D scene.

- Surgical instruments can be tracked and visualized in the 3D scene of the patient's anatomy.

The $3 D$ Slicer implementation uses primarily the " $V$ isualization Toolkit" library (VTK, [7]) and the "Tool Command Language/graphical user interface Toolkit" scripting language ( $\mathrm{Tcl} / \mathrm{Tk},[\underline{8})$. We used the same programming environment and simply added the necessary VTK-filters and Tcl-commands.

\subsection{Simulation}

To simulate the intervention, we combined needle-like VTK-objects representing the probes with VTK-objects representing the frozen or ablated tissue (Figure 11. The radiologist can add these models into the virtual scene of the 3D Slicer in which the segmented and grayscale data of the patient are also visualized. A powerful visualization is therefore straightforward and, depending on the specific case, it is possible to place any number of probes into the virtual 3D scene (Figure B1).

The virtual probes: So far we have implemented a cryo-probe consisting of one single needle (Figure $\mathbb{T}_{\mathrm{H}}$ ), a radiofrequency probe with one needle and a radiofrequency probe with three needles, called a cluster (Figure $\$$ ). As $3 D$ Slicer uses a world coorindate system in millimeter-space, the parameters for the virtual probes are simply given by the parameters of the real probes. This also facilitates the modification of specific simulation-parameters or the addition of other models to the list of virtual probes according to technological and scientific progress.

The frozen, or ablated, tissue: To get a reasonable representation of killed tissue during cryo-ablation, we used the following hypothesis that has historically also guided radiologists carrying out interventions:

Hypothesis: Apart from the $0.5 \mathrm{~cm}$ outer rim of the formed iceball, all the cells inside the frozen region are killed during cryo-therapy.

The outer cells are simply lying too far away from the cooling source, implying too high temperatures for cell death. Two important statements are equivalent to this hypothesis. First, the shape of the zone representing the killed tissue is the same as the shape of the formed iceball, but smaller. Secondly and equivalently, the radiologist should freeze an additional $0.5 \mathrm{~cm}$ margin around the tumor to kill all the cancerous cells. We decided to take the second point of view and to simulate the iceballs during cryo-therapy and not directly the killed tissue, but 
a)

b)
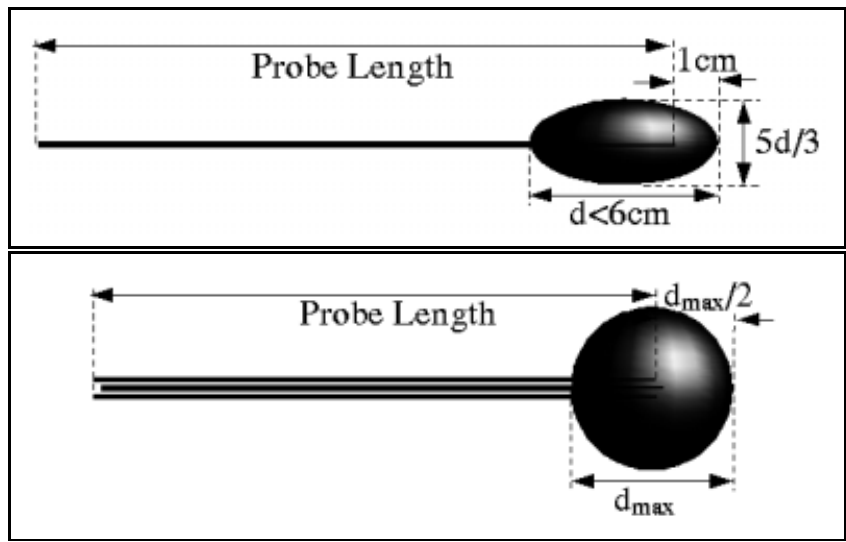

Fig. 1. In a) we show a virtual cryo-probe including the elliptic iceball and a needle. The image in b) shows a radiofrequency cluster consisting of three needles and a spherical ablation model.

to require the coverage of an additional $0.5 \mathrm{~cm}$ margin around the tumor. This approach is more compatible to MR guidance, as MR images can visualize the iceballs but not the ablated tissue directly.

In order to visualize the additional $0.5 \mathrm{~cm}$ margin around the tumor, we implemented a VTK filter that dilates the segmented tumor. The result is then added at the appropriate position to the 3D-scene of the $3 D$ slicer.

We also aimed to simulate the formation of the iceball during cryo-therapy. 4 ] and [5] have performed studies to describe the iceball-shape during cryo-ablation. Their results as well as our own studies have shown elliptic to teardrop-shaped iceballs for a single probe. Therefore we used ellipsoids to model the frozen zones at the tip of each needle (Figure 1 a). The specific parameters were assessed from MR images of several previous cryo-cases showing the iceballs at the end of the freezing cycle. The specific parameters vary for different probes, as e.g. the iceball size depends on the cooling rate and therefore on the probe diameter.

For radiofrequency ablation, we simulate directly the killed tissue and therefore no additional margin has to be simulated. The appropriate model for the ablated zone is spheres, as indicated by previous radiofrequency cases (Figure 10).

\subsection{Optimization}

As well as the visual verification of a simulated probe setup, we also provide a more objective quality measure. Such a medically relevant measure has to reflect the following two central factors:

1. How much of the tumor is ablated with a chosen setup. Therefore we have to calculate the percentage of the tumor (including the additional $0.5 \mathrm{~cm}$ margin) that would be lying within the simulated iceballs. 
2. Furthermore it's important to know how much healthy tissue would also be killed with a specific treatment plan. This is done by calculating the volume of the simulated iceball that is not covering cancerous but instead healthy tissue.

A good setup would maximize the first value (the volume of ablated cancerous tissue) and minimize the second (the volume of killed healthy cells). We developed a mathematical expression that reflects these factors and allows the radiologists to use a semi-automated setup-optimization and selection.

Let $V_{1}$ denote the volume of killed healthy tissue, $V_{2}$ denote the volume of ablated tumor and $V_{\text {tumor }}$ denote the volume of the whole tumor $\left(V_{1}, V_{2}\right.$ and $V_{\text {tumor }}$ in $\mathrm{ml}$ ). $\boldsymbol{x}$ is the vector that represents the positions and orientations of the probes as well as the size of the frozen iceballs at their tips. We can then define the following measure:

$$
I(\boldsymbol{x})=\lambda \cdot \frac{V_{1}(\boldsymbol{x})}{V_{\text {tumor }}}-(1-\lambda) \cdot \frac{V_{2}(\boldsymbol{x})}{V_{\text {tumor }}}+\left(1-\delta_{\boldsymbol{x}, \Omega}\right) \cdot \frac{V_{\text {tumor }}}{m l}
$$

where we have simply

$$
\Omega=\left\{\boldsymbol{x} \in \mathbf{R}^{n} \mid \mu_{i} \leq x_{i} \leq \sigma_{i} ; \mu_{i}, \sigma_{i} \in \mathbf{R}\right\}
$$

and

$$
\delta_{\boldsymbol{x}, \Omega}=\left\{\begin{array}{l}
1, \text { if } \boldsymbol{x} \in \Omega \\
0, \text { if } \boldsymbol{x} \notin \Omega
\end{array}\right.
$$

The potentially optimal setup is found by looking for the vector $\boldsymbol{x}$ that minimizes the measure $I(\boldsymbol{x}) . \lambda$ is a very important factor that weighs the two somewhat contradictory terms of minimizing $V_{1}$ and maximizing $V_{2}$. $\lambda$ has to be chosen carefully, depending on the specific medical application. For example in brain tumor ablation, it's very important to minimize the ablated healthy tissue, so we would choose a $\lambda$ close to 1 . On the other hand, $\lambda$ can lie much closer to 0 for liver tumors. $\Omega$ defines the bounds of the admissible optimization space and should describe an anatomically possible window for probe trajectories (e.g. we can't pass through any bones, even if the tumor ablation would be more optimal for such a setup).

The optimization algorithm uses primarily a VTK filter for boolean subtraction and reunion of polygonal datasets, a second VTK filter to calculate volumes of polygonal datasets and the "Powell" optimization algorithm from [6].

The combination of visualization, simulation and optimization results in an interactive planning tool for minimally invasive tumor ablation. In practice the radiologists can drag and rotate the probes (so far cryo- or radiofrequency probes) into an initial setup within the patient's segmented anatomy and then start the optimization algorithm to improve the probes' placement. The interventional radiologist can also get an objective quality measure to decide if e.g. an additional probe should be used to increase the likelihood of complete tumor ablation. 
a)

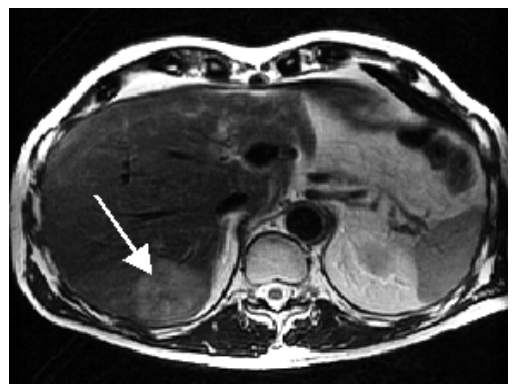

b)

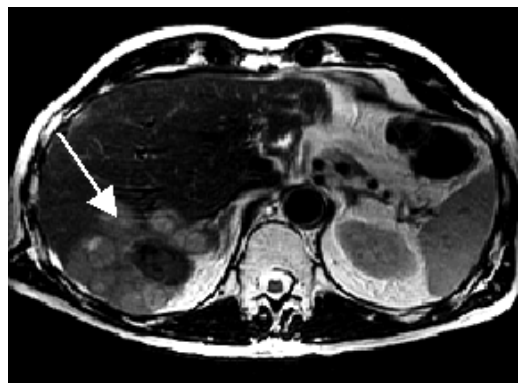

Fig. 2. In figure a) we show the tumor (arrow) in a pre-operative T2-weighted FSE. In b) we can see the ablated area as a dark, ellipsoidal zone. But we also recognize that during the last three months, an outer rim of cancerous cells (arrow) grew around the initial tumor.

\section{Results}

We applied our planning tool to analyze the failure of a previously performed cryo-therapy case. In Figure 2a we see an axial slice of the pre-operative T2weighted fast spin-echo (FSE) MR scan of the abdomen. The arrow points at the tumor. In $2 \mathrm{~b}$, a slice of the 3-month follow-up scan (same modality) is shown. Unfortunately the tumor had re-appeared (arrow) and had grown very quickly around the effectively ablated zone. The analysis of this case was very important as the radiologists were convinced of having completely frozen the whole tumor including the additional $0.5 \mathrm{~cm}$ margin. In consequence, some concerns about the frequently used hypothesis above arose. So we checked if, because of the failure of this treatment, the hypothesis has effectively to be questioned and, if not, to propose a safer treatment plan.

\subsection{Simulating the Treatment Plan}

In Figure $3 \mathrm{a}$ and b, we simulate the setup that was chosen by the radiologists. This is easily possible as the treatment was performed under near-realtime MR guidance and interventional MR scans enable the visualization of the probes and of the growing iceballs.

During pre-operative planning such a 3D view indicates if, for a certain setup, the probes are getting too close to important anatomical structures, such as main vessels or the gallbladder. Furthermore, a chosen probe might be too short to effectively reach its predetermined position in the tumor, and the radiologist can decide to take a longer probe.

In a next step, we wanted to visualize the simulation of ablated tissue as described in section Simulation. This is shown in Figure $4 a$, where the simulated iceball of the chosen setup is in red and the tumor, including the $0.5 \mathrm{~cm}$ margin, in brown. Volume calculations indicate that just about $63 \%$ of the necessary volume are frozen in this setup. To increase this rather poor value, we applied 
our optimization algorithm for this setup. The frozen volume increases to $74 \%$ of the tumor volume (including margin).

A visual interpretation of Figure 4 indicates that the tumor was undertreated with the chosen probe setup. The calculated percentage of killed cancerous tissue $(63 \%)$ confirms this estimation. Even after optimization, the ablation would still be insufficient (74\%). Therefore the use of more cryo-probes is suggested.

\subsection{Improving the Treatment Plan}

In order to increase significantly the percentage of ablated cancerous tissue, we had to add two more probes to the scene. In Figures $3 \mathrm{k}$, d and Figure $4 \mathrm{~b}$, we show the results. For this setup and after optimization, our measure indicated that $92 \%$ of the necessary volume would be frozen. The new setup would have increased the likelihood of successful tumor ablation for this patient.

\subsection{Checking the Iceball Simulation}

To check the quality of our iceball simulation, we superimposed the simulation and the real iceball that was segmented from the intra-operative MR scan at the end of the freezing cycle. The result is shown in Figure 4r (real iceball is shown in white and translucent, and the simulated iceball in red).

The ellipsoids simulate nicely the rough shape of the real iceball. But smaller details of the frozen region are not present in our estimation.

\subsection{Post-operative Analysis}

At the end of the freezing cycle, the radiologist can check if the performed treatment has most probably been successful. Such an analysis estimates also the quality of our simulation and checks its predictions. We superimposed the segmented iceball at the end of the freezing cycle with the tumor. The result is shown in Figure $4 \mathrm{~d}$, and re-confirms the result of our simulation: the tumor has been undertreated.

\section{Discussion}

The planning tool described here was able to explain the failure of a cryo-therapy case. In particular, even though the hypothesis above has still not been proven to be correct, its possible incorrectness can not be inferred from the failure of the presented case. Our tool will help to check and, if necessary, to reformulate this very important hypothesis.

We have also presented the different capabilities of the software to minimize the risk of failure:

- Within the visualization package $3 D$ Slicer, we can provide easily interpretable 3D visualizations of the patients' anatomy. 
- Virtual needles and iceballs can be added to the virtual scene. This can ensure the safety of specific insertion directions of the probes and a good choice of the probe (e.g. its length). It improves also the visual quality estimation of a specific setup.

- The addition of an objective quality measure and a semi-automated optimization algorithm minimizes the danger of failure.

With these features, the tool was also able to propose a better probe setup which would have increased significantly the likelihood of success.

There are also some very important improvements necessary to make this tool widely used:

- We are using the models of Figure 1 to simulate the ablated volumes. These rough estimates are based on a limited number of cases and should be refined in the future. For example, the use of several probes does not necessarily result in the same iceball as the union of several single-probe iceballs.

- The optimization algorithm should be improved. For example a parallel implementation would significantly reduce calculation time. So far it takes up to 15 minutes on a $440 \mathrm{MHz}$ UltraSPARC-IIi Ultra 10 Workstation with $512 \mathrm{MB}$ RAM to optimize the placement of two cryoprobes (presented case). It is also desirable to determine the optimization space $\Omega$ (Equation 2) through the anatomical objects in the virtual scene and not explicitly by the radiologists.

- It would be very desirable to combine the tool with a non-rigid registration algorithm ([9]), so that the pre-operative planning can be adjusted to the patient's position in the interventional MR ([11, [10]).

\section{Conclusion}

The results of this analysis underline the importance of a planning tool having similar functionalities as the presented software. We have shown that this tool has a significant potential to help interventional radiologists before and during image guided interstitial tumor ablation. We have concentrated upon cryotherapy, but other medical applications would be radiofrequency-, laser- or focused ultrasound ablation. The implementation can be done in complete analogy to cryotherapy, and in fact, radiofrequency has already been added to the presented package.

We are convinced that the combination of the presented planning tool with segmentation, non-rigid registration and interventional imaging, has the potential to improve significantly the quality of image guided percutaneous tumor ablation. The general aproach of pre-operative simulation and automated optimization has an even wider range of potential applications. For example for brachytherapy similar approaches have already been developed ([12]) and are used successfully. 
a)

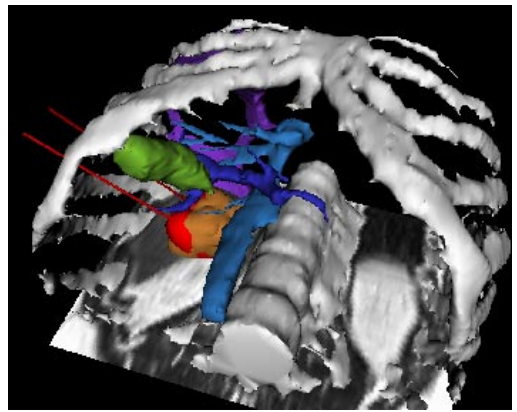

c)

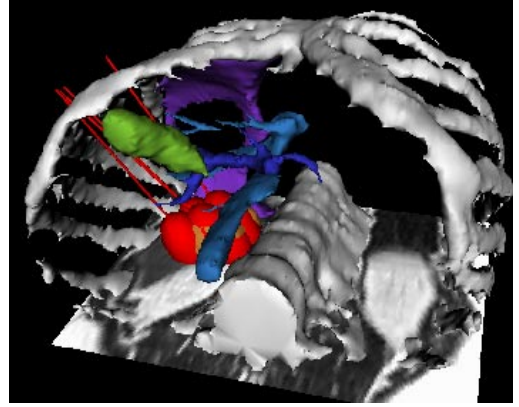

b)

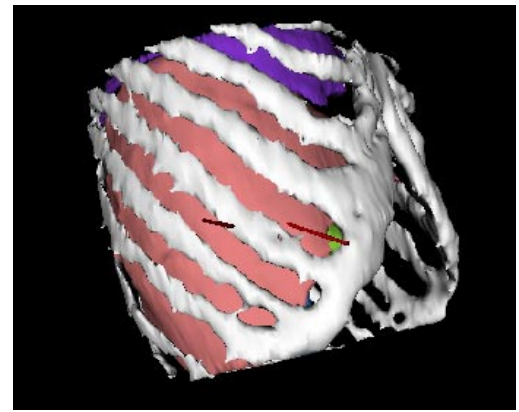

d)

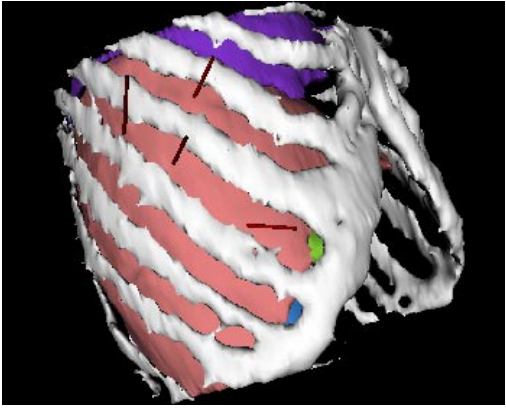

Fig. 3. The virtual cryo-probes, simulating the the needles and the frozen tissue (shown in red), can be set into spatial correspondence to the patient's segmented anatomy and the corresponding intensity scans. The tumor and an additional $0.5 \mathrm{~cm}$ security margin, is shown in brown. The main vessels are in blue, the gallbladder in green and the bones in white. a) and b) show the setup chosen by the radiologists for this specific case, while we present a better setup which would have decreased significiantly the danger of undertreating the cancer in images c) and d).

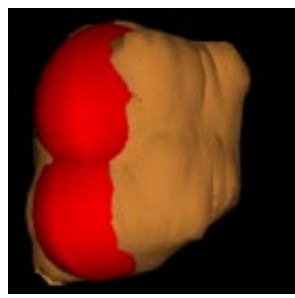

a)

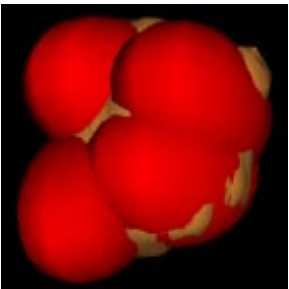

b)

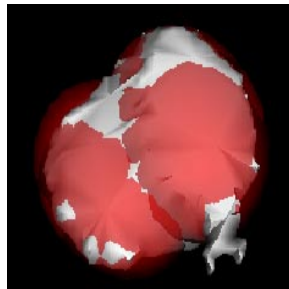

c)

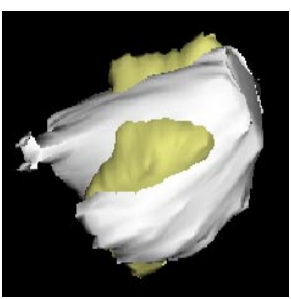

d)

Fig. 4. In a) we show the virtual iceballs (red) according to the real treatment plan. This $3 \mathrm{D}$ visualization would indicate undertreatment of the tumor (tumor, including margin, is shown in brown). Volume calculations estimate that only about $63 \%$ of the tumor would be killed. In b) we propose a setup that increased the percentage of killed cancerous tissue to about $92 \%$ of the whole tumor volume. In image c) we compare our iceball simulation (red) to the real iceball (white and translucent) and see that the simulation is pretty good, though not perfect. In d), we have superimposed the tumor (yellow) and the real iceball at the end of the freezing cycle (white). Even without the $0.5 \mathrm{~cm}$ margin, the tumor has not been completely frozen. 


\section{References}

1. F.A. Jolesz; Image-Guided Procedures and the Operating Room of the Future; Radiology, 204(3):601-612, May 1997.

2. S.G. Silverman, K. Tuncali, D.F. Adams, et al.; Percutaneous MR Imaging-guided Cryotherapy of Liver Metastases; Radiology, 213:122, November 1999.

3. D. Gering; A System for Surgical Planning and Guidance Using Image Fusion and Interventional MR; MIT Master's Thesis, 1999

4. J.C. Saliken, J. Cohen, R. Miller and M. Rothert; Laboratory Evaluation of Ice Formation around a 3-mm Accuprobe; Cryobiology, 32:285-295, 1995.

5. C.M. Lam, S.M. Shimi and A. Cuschieri; Thermal Characteristics of a Hepatic Cryolesion Formed in Vitro by a 3-mm Inplantable Cryoprobe; Cryobiology, 36:156164, 1998.

6. W.H. Press, S.A. Teukolsky, W.T. Vetterling and B.P. Flannery; Numerical Recipes in C (Second Edition); Cambridge University Press, 1992.

7. W. Schroeder, K. Martin and W. Lorensen; The Visualization Toolkit: An Objectoriented Approach to 3D Graphics; Cambridge University Press, 1992.

8. B.B. Welch; Practical Programming in Tcl and Tk; Prentice Hall PTR, 1997.

9. S.K. Warfield, M. Kaus, F.A. Jolesz and R. Kikinis; Adaptive, Template Moderated, Spatially Varying Statistical Classification; Medical Image Analysis, 4(1):43-55, 2000.

10. M. Ferrant, S.K. Warfield, C.R.G. Guttmann, R.V. Mulkern, F.A. Jolesz and R. Kikinis 3D Image Matching Using a Finite Element Based Elastic Deformation Model; MICCAI 1999, pp. 202-209.

11. Simon K. Warfield, Arya Nabavi, Torsten Butz, Kemal Tuncali, Stuart G. Silverman, Peter McL. Black, Ferenc A. Jolesz, and Ron Kikinis; Intraoperative Segmentation and Nonrigid Registration For Image Guided Therapy; MICCAI 2000, accepted to appear.

12. E.K. Lee, R.J. Gallagher, D. Silvern, Ch.-S. Wuu and M. Zaider; Treatment Planning for Brachytherapy: An Integer Programming Model, two Computational Approaches and Experiments with Permanent Prostate Implant Planning; Phys. Med. Biol., 44(1):145-165, 1999. 